\title{
Conservative parabolic problems: non-degenerated theory and degenerated examples from population dynamics
}

\author{
Olga Danilkina, Max O. Souza ${ }^{\dagger}$ Fabio A. C. C. Chalub
}

December 5, 2017

\begin{abstract}
We consider partial differential equations (PDE) of drift-diffusion type in the unit interval, supplemented by either two conservation laws or by a conservation law and a further boundary condition. We treat two different cases: (i) uniform parabolic problems; (ii) degenerated problems at the boundaries. The former can be treated in a very general and complete way, much as the traditional boundary value problems. The latter, however, brings new issues, and we restrict our study to a class of forward Kolmogorov equations that arise naturally when the corresponding stochastic process has either one or two absorbing boundaries. These equations are treated by means of a uniform parabolic regularisation, which then yields a measure solution in the vanishing regularisation limit. Two prototypical problems from population dynamics are treated in detail. For these problems, we show that the structure of measure-valued solutions is such that they are absolutely continuous in the interior. However, they will also include Dirac masses at the degenerated boundaries which appear, irrespective of the regularity of the initial data, at time $t=0^{+}$. The time evolution of these singular masses is also explictly described and, as a by-product, uniqueness of these measure solution is obtained.
\end{abstract}

\footnotetext{
*Department of Mathematics, College of Natural and Mathematical Sciences, the University of Dodoma, P.O. BOX 259,

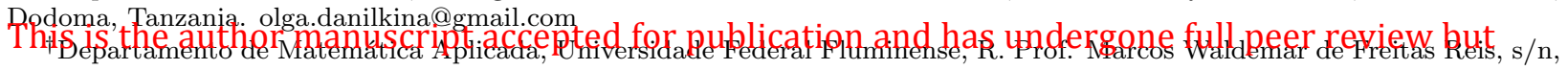

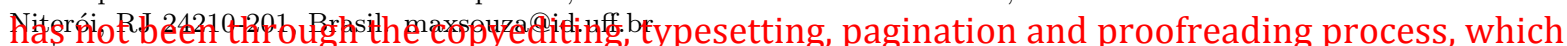
* Departamento de Matemática and Centro de Matemática e Aplicações, Universidade Nova de Lisboa, Quinta da Torre,

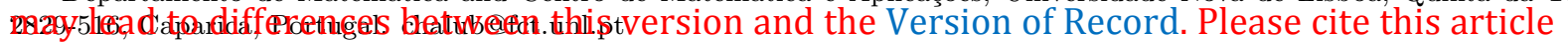

as doi: 10.1002/mma.4901 


\section{Introduction}

\subsection{Background}

Partial differential equations (PDE) are an ubiquitous tool for modelling a variety of phenomena in the applied sciences. Typically, a PDE in any given domain will not be well-posed, at least in the sense of uniqueness, unless further assumptions are made on the solutions. Such assumptions can range from traditional boundary conditions in bounded domains - eg. Dirichlet or Neumann — to integrability conditions in unbounded domains, and might include a mixture of both - as for instance, in the case of degenerated equations in part of the boundary. This is the traditional state of affairs as presented in most of the classical introductions to the subject $[32,35,38,49]$.

From a different perspective, a number of these models are derived from integral formulations that arise naturally from conservation laws that are expected to hold in the problem being modelled. This is well known in the literature of conservation laws in hyperbolic problems - cf. [20] — but it also appears in other settings within hyperbolic problems: $[7,46,47]$. In the context of parabolic operators, however, this class of problems seems to have received much less attention.

A very similar class of problems, though, has received somewhat more attention: the solution of parabolic operators with the specification of an integral constraint and a boundary condition. The study of these problems seems to date back at least to $[9,22]$, who studied specific problems in this class for the heat equation. A proof of existence and uniqueness for a problem in this class, with a general linear parabolic operator can be found already in [39]. The subject has resurfaced from the late seventies to the early nineties with the works of $[10,11,36,37,48,53]$.

Beginning in the late nineties, there is a growing interest in understanding the solutions of the heat equation subject to the specification of the first two linear moments, which seems to be first addressed by [4], but also include [5,6,21]. Numerical methods for these problems were also developed in a series of works [8,23]. More recently, using a combination of variational and semigroup methods, [44] presents a very detailed theory for the heat and wave equation in this setting. See also [43] for companion results to diffusive equations with the $p$-Laplacian. Further discussion and references can be found in [44]. See [45] for a discussion on conservation laws of $1+1$ linear parabolic equations from the point of view of Lie symmetries.

The number of linear conservation laws that can be imposed, while still leading to a well posed problem, is also of relevance; in [43] a full family of moments $\mu_{n}(f)=\int(1-x)^{n} f \mathrm{~d} x$ is considered. However, well posedness of the problem requires that at most two of them are prescribed. We are not aware of any wellposed linear problem, in one variable, where more than two linearly independent conservation laws were prescribed. For the 2D case, however, [25] imposes 4 linear conservation laws.

It is clear that conservation laws are a particular case of integral conditions that might be specified, in lieu of one or more boundary conditions, to the solution of certain PDEs; see, e.g. [24,26] for problems that specify one classical boundary condition plus imposing one time-dependent integral relationship.

In this work we are interested, as discussed above, in linear equations with associated linear conservation laws. However, the field of non-linear equation with non-linear conservation laws is also a topic of interest. This includes celebrated equations and systems, as the Korteveg-de Vries (KdV) equation and the AKNS system, where an infinite number of independent conservation laws can be imposed. For an introduction to the topic, see [28].

From now on, it will be always implied that all conservation laws of interest are linear.

As far as conservation laws for degenerated parabolic problems are concerned, the earliest work that we are aware of is due to the two last authors in [13], which shows that a degenerated parabolic equation the so-called generalised Kimura equation from population genetics - subject to two conservation laws namely the conservation of probability and conservation of centering with respect to the fixation probability - is well posed in the space of Radon measures. A generalisation of this problem to higher dimensions 
in the context of the Wright-Fisher process is given in [16], and a study of the PDE version for the SIS epidemiological model which is degenerated at the origin and needs a boundary condition at 1 is given in [17]. A recent work showing that the heat equation subject to conservation of the first two moments is well posed in all $L^{p}$ spaces for $p \geq 1$, and even in $C^{0}$, can be found in [3].

\subsection{Some degenerated Fokker-Planck equations from population dynamics}

The ultimate goal of this work is to understand how the solution of parabolic conservative problems, which are degenerated at least in one of the boundaries, can be approximated by the solutions of non-degenerated problems. As a by-product, we will be able to characterise a condition that guarantees positiveness of the solution obtained. It will also allow to obtain small test function spaces where existence and uniqueness of the solution hold.

In what follows, we shall focus on two examples. Our first example is the so called Generalised Kimura Equation [13]:

$$
\partial_{t} u=\partial_{x}^{2}(x(1-x) u)-\partial_{x}(x(1-x) \psi(x) u)
$$

where $(x, t) \in[0,1] \times \mathbb{R}_{+}$and $\psi:[0,1] \rightarrow \mathbb{R}$ is a function in a space to be defined latter on (known as fitness in the biological literature). The initial condition is given by

$$
u^{(\mathrm{I})}=u(\cdot, 0) \geq 0, \quad x \in[0,1],
$$

and the solution of this equation should obey two integral conditions (conservation laws):

$$
\begin{aligned}
& \frac{\mathrm{d}}{\mathrm{d} t} \int_{0}^{1} u \mathrm{~d} x=0 \\
& \frac{\mathrm{d}}{\mathrm{d} t} \int_{0}^{1} \varphi(x) u \mathrm{~d} x=0
\end{aligned}
$$

where $\varphi:[0,1] \rightarrow \mathbb{R}_{+}$is the unique solution of

$$
\varphi^{\prime \prime}+\psi(x) \varphi^{\prime}=0, \text { with } \varphi(0)=0 \text { and } \varphi(1)=1 \text {, i.e. } \varphi(x)=\frac{\int_{0}^{x} \mathrm{e}^{-\int_{0}^{y} \psi(z) \mathrm{d} z} \mathrm{~d} y}{\int_{0}^{1} \mathrm{e}^{-\int_{0}^{y} \psi(z) \mathrm{d} z} \mathrm{~d} y} .
$$

Equation (1) was formally derived in [40], for the constant fitness case, and extensively studied in [30, $33,34]$. Its first rigorous derivation, as the large population limit of the Wright-Fisher process, appears in [31]. More recently rigorous derivations, starting from various stochastic processes and more general fitness functions, can be found in [18] and [13]. In the latter, it was shown that the correct solution from the modelling point of view - satisfies two conservation laws. Such a generalised Kimura equation was extensively studied in [14], where the measure nature of the solutions was first obtained. See also [12] for an earlier connection with solutions of the Replicator Dynamics and [16] for generalisations to higher dimensions. Similar equations, but with slightly different conditions, were also studied in $[15,17]$ — see below. See also [29] for a comprehensive treatment using classical tools. A similar equation was investigated in [52], where mutations were allowed and hence boundaries were not absorbing. In this case, appropriate local non-flux condition could be imposed.

Our second example comes from epidemiology: the SIS-PDE model that is given by

$$
\partial_{t} p=-\partial_{x}\left\{x\left[R_{0}(1-x)-1\right] p\right\}+\frac{1}{2} \partial_{x}^{2}\left\{x\left(R_{0}(1-x)+1\right) p\right\}
$$


satisfying the boundary condition

$$
\left[\frac{1}{2}\left[\left.\left(1-R_{0}\right) p\right|_{1}+\left.\partial_{x} p\right|_{1}\right]+\left.p\right|_{1}\right]=0
$$

and the conservation law

$$
\frac{\mathrm{d}}{\mathrm{d} t} \int_{0}^{1} p(x, t) \mathrm{d} x=0 .
$$

This model is an intermediate model in between the Markov process associated to the Susceptible-InfeciousSusceptible epidemiological model and its ODE counterpart. See [17] for further details.

Note that equations (1) and (6) are degenerated Fokker-Planck equations, for which no boundary condition can be imposed in the degenerated boundary, other than integrability. These equations are associated to diffusion approximation of Markov chains with absorbing states, and are studied, for instance, in [27]. Applications to biology in a framework similar to ours can be found in $[29,30,33,34]$.

\subsection{Summary of results and outline}

We begin in Section 2 by defining a non-degenerated conservative parabolic problem, and the first result shows that the conservative problem can be converted to a coupled boundary value problem, and that the conservations laws must be related to the kernel of the formal adjoint. The next step is then to define non-degenerated positive problems in terms of the conservation laws. For a very general class of positive problems, we are able to show existence and uniqueness along the same lines of the more classical boundary conditions, by recurring to general Sturm-Liouville theory. In particular, if the coefficients of the equations are smooth, we obtain that the solutions are $C^{\infty}$. This Section can be seen as an extension of the theory presented in [44].

In Section 3 we start the study of our degenerate problems. We introduce a class of self-adjoint elliptic perturbations, which are then readily amenable to be treated by the theory developed in the previous Section. It turns out that the solutions can be naturally treated as measures, and Prokohorov theorem can be used to pass to the limit as the perturbation vanishes [2]. This very weak solution then satisfies all the required conditions. A further analysis of the solution is presented in Section 4, where we consider two different problems that arise from population dynamics. Their solutions are absolutely continuous in the interior, but turn out to contain Dirac masses in the degenerated boundaries. The absolutely continuous part is a classical solution of the corresponding PDE, and the singular masses at the endpoints appear at $t=0^{+}$, irrespective of the regularity of the initial condition. Moreover, a description of the evolution of these singular masses is also given - in particular, for sufficiently regular coefficients, the evolution of the singular measures is given by time integrals of boundary values of the absolutely continuous part of the solution. As a by-product of this description, we show uniqueness of such measure solutions. Finally, optimal domains of test functions for the weak solutions are also discussed.

We close this work with a discussion of the results presented in Section 5.

\section{Conservative parabolic problems}

We begin by considering uniformly parabolic problems in self-adjoint form. To this end, let

$$
L v=\partial_{x}\left(p(x) \partial_{x} v\right)+q(x) v
$$

defined in some finite interval $I=(a, b)$, and with $p>0$, and with $p, q \in L^{2}(I) \cap L^{\infty}(I)$. 
Definition 1. A (totally) conservative parabolic problem is an initial value problem of the form:

$$
\left\{\begin{array}{lr}
\partial_{t} v=L v, & \text { in I and } t>0 \\
\frac{\mathrm{d}}{\mathrm{d} t}\left\langle v(\cdot, t), \phi_{1}\right\rangle=0, & t>0 \\
\frac{\mathrm{d}}{\mathrm{d} t}\left\langle v(\cdot, t), \phi_{2}\right\rangle=0, & t>0 \\
v(x, 0)=v_{0}(x) &
\end{array}\right.
$$

where $\langle\cdot, \cdot\rangle$ denotes the inner product in $L^{2}(I)$ and $\phi_{1}, \phi_{2} \in L^{2}(I)$ are not multiple of one another. The conditions $\frac{\mathrm{d}}{\mathrm{d} t}\left\langle v(\cdot, t), \phi_{i}\right\rangle=0, i=1,2$ will be termed (linear) conservation laws. From now on, we will assume that all conservation laws (CLs) considered here are linear, and hence we will refer to them only as $C L s$.

If the infinitesimal generator associated to Equation (9) is self-adjoint, then it turns out that the possible choices for $\phi_{1}$ and $\phi_{2}$ are limited:

Theorem 1. The operator $L$ in (9) can be taken to be self-adjoint if, and only if, $\phi_{1}$ and $\phi_{2}$ are linearly independent solutions of the $O D E L v=0$, and if problem (9) can be recast as a coupled (non-local) boundary value problem as follows

$$
\left\{\begin{array}{lr}
\partial_{t} v=L v, & \text { in } I \text { and } t>0 \\
p(b)\left[\partial_{x} v(b, t) \phi_{1}(b)-v(b, t) \phi_{1}^{\prime}(b)\right]-p(a)\left[\partial_{x} v(a, t) \phi_{1}(a)-v(a, t) \phi_{1}^{\prime}(a)\right]=0, & t>0 \\
p(b)\left[\partial_{x} v(b, t) \phi_{2}(b)-v(b, t) \phi_{2}^{\prime}(b)\right]-p(a)\left[\partial_{x} v(a, t) \phi_{2}(a)-v(a, t) \phi_{2}^{\prime}(a)\right]=0, & t>0 \\
v(x, 0)=v_{0}(x) &
\end{array}\right.
$$

Furthermore, we have that $\phi_{1}$ and $\phi_{2}$ are eigenfunctions of $L$ associated to the eigenvalue $\lambda=0$.

Proof. Assume $L$ is self-adjoint with domain $D(L)$. Then the conservation conditions are equivalent to

$$
\left\langle\varphi, L \phi_{i}\right\rangle=0, \quad i=1,2 ; \quad \varphi \in D(L)
$$

Since $L$ is self-adjoint, it is densely-defined, and hence the identities above hold on $L^{2}(I)$. Therefore, we have that $L \phi_{i}=0, i=1,2$. Since they are not multiple of one another, they are linearly independent solutions 
of $L v=0$. Also, under the assumptions on the coefficients of $L$, we have that any solution to the ODE is of class $C^{1}$. Thus, since $L$ is self-adjoint, direct integration by parts yields (10).

Conversely, assume that problem (9) is equivalent to problem (10). Then a direct computation shows that $L$ is symmetric. Since $\phi_{1}, \phi_{2}$ are solutions of $L v=0$, it follows immediately from Proposition 2.1 in [1] - see also $[41,51]$ — that $L$ can be taken to be self-adjoint.

For the last claim, we already have that $L \phi_{i}=0$, for $i=1,2$. Therefore, it remains to show that every $\phi_{i}$ satisfies the boundary conditions. We shall check this for $\phi_{1}$ - the case of $\phi_{2}$ is analogous. The first condition is easily verified by direct substitution of $\phi_{1}$ into $v$. In order to verify the second condition, we write $B_{2}$ for its left hand side and compute:

$B_{2}=p(b)\left[\phi_{1}^{\prime}(b) \phi_{2}(b)-\phi_{1}(b) \phi_{2}^{\prime}(b)\right]-p(a)\left[\phi_{1}^{\prime}(a) \phi_{2}(a)-\phi_{1}(a) \phi_{2}^{\prime}(a)\right]=p(b) W_{\left\{\phi_{1}, \phi_{2}\right\}}(b)-p(a) W_{\left\{\phi_{1}, \phi_{2}\right\}}(a)=0$ where $W_{\left\{\phi_{1}, \phi_{2}\right\}}(x)$ is the Wronskian of functions $\phi_{1}$ and $\phi_{2}$ at $x$ and, finally, the last equality follows from Abel's theorem.

Remark 1. The proof of Theorem 1 also shows that, for self-adjoint problems, any conservation law, in the sense of Definition 1, must be a solution of the second order ODE Lv =0, and hence that only two linearly-independent conservation laws might be imposed in such a problem. Similar restrictions also appear when considering other problems: there are counterexamples which show that not every choice of conserved moments yields a well posed problem for the porous media equation - cf. [50]. However, the choice of the Oth order moment (mass conservation) and a further higher order moment may yield a well posed problem as shown in [43].

Definition 2. We say that (10) is a non-negative conservative problem, if we have a conservative problem with $\phi_{1}$ and $\phi_{2}$ being non-negative. If, in addition, there exists at least one solution of the ODE Lv $=0$ that is positive everywhere then we say that the problem is intrinsically positive.

Lemma 1. Assume that we have an intrinsically positive conservative problem, and consider the correspond- 
ing spectral problem $-L u=\lambda u$, with coupled boundary conditions. Then, we can order the eigenvalues such that we have $0=\lambda_{1}=\lambda_{2}<\lambda_{3} \leq \cdots \lambda_{n} \leq \lambda_{n+1} \leq \cdots$.

Proof. Since $p>0$, we have that the set of eigenvalues is bounded from below and and is unbounded from above. In particular, we can order the eigenvalues such that $\lambda_{1} \leq \lambda_{2} \leq \lambda_{3} \leq \cdots \lambda_{n} \leq \lambda_{n+1} \leq \cdots$. From Theorem 1, we already know that zero is an eigenvalue of multiplicity two. Since the problem is intrinsically positive, we have a choice of two linear independent eigenfunctions that are positive everywhere. From the oscillatory theory of Sturm-Liouville problems [51, Theorem 13.5], if we have $\lambda_{n}=\lambda_{n+1}$ then the corresponding eigenfunctions have either $n-1$ or $n$ zeros. Thus, if $\lambda=0$ is not the principal eigenvalue, any eigenfunction associated to it must have at least one zero in the interior, and this is not possible for an intrinsically positive problem. Hence $\lambda_{1}=\lambda_{2}=0$. Since the problem is second order, the eigenvalues can have multiplicity at most two, and hence $\lambda_{3}>0$.

Remark 2. Recall that if $p, q \in L^{2}(I)$ then the eigenfunctions are in $H^{2}(I)$. In general, if $p, q \in C^{k}(I)$, then the eigenfunctions will be of class $C^{k+2}-c f .[19,54]$.

In what follows, we shall assume that $p$ and $q$ are given, and for an intrinsically positive problem we shall write $w_{1}=\psi_{1}, w_{2}=\psi_{2}$, where $\psi_{1}, \psi_{2}$ are eigenfunctions corresponding to the zero eigenvalue, with unit norm, and that are positive everywhere. Furthermore, the set $\left\{w_{k}, k \geq 3\right\}$ will correspond to the unit eigenfunctions associated to the positive eigenvalues.

Before we state the next result, we introduce the following harmonic spaces — cf. [49]: for non-negative $s$, we define

$$
\left.\mathcal{H}_{s}=\left\{f \in L^{1}(I) \text { s.t. } \sum_{k=0}^{\infty} \hat{f}(k) \lambda_{k}^{s / 2} w_{j} \in L^{2}(I)\right\}, \quad \hat{f}(k)=\left\langle f, w_{k}\right)\right\rangle,
$$

for $s \in \mathbb{R}$. Notice that

$$
\|f\|_{s}^{2}=\sum_{k=1}^{\infty}|\hat{f}(k)|^{2} \lambda_{k}^{s}
$$

and that we have $\mathcal{H}_{0}=L^{2}(I)$ and $\mathcal{H}_{1}=H^{1}(I)$. In addition, we have that $\mathcal{H}_{-s}$ is the dual of $\mathcal{H}_{s}$, and that the Fourier series characterisation is still valid.

Theorem 2. Consider the initial value problem (10) and assume that it is intrinsically positive. If we have $v_{0} \in L^{2}(I)$, then, for any $T>0$, there exists a unique solution in the class $C\left([0, T) ; L^{2}(I)\right) \cap C^{\infty}\left((0, T) ; \mathcal{H}_{1}\right)$. Furthermore, if $v_{0} \geq 0$, then we have $v(\cdot, t) \geq 0$ for every $t$. 
Proof. The existence proof is standard, and we have that

$$
v(x, t)=\sum_{k=1}^{\infty} a_{k} \mathrm{e}^{-\lambda_{k} t} w_{k}, \quad a_{k}=\left\langle v_{0}, w_{k}\right\rangle .
$$

Before we discuss uniqueness, we shall investigate the positiveness of the solutions. In order to show the positiveness of a given solution at any time, we first assume that $v_{0}>0$, and that $p$ and $q$ are smooth. In this case we have that $v \in C\left([0, T) ; L^{2}(I)\right) \cap C^{\infty}\left((0, T) ; C^{\infty}(I)\right)$.

Let

$$
v_{s}=a_{1} w_{1}+a_{2} w_{2} \text { and } v_{t}=\mathrm{e}^{-\lambda_{3} t} \sum_{k=3}^{\infty} a_{k} \mathrm{e}^{-\left(\lambda_{k}-\lambda_{3}\right) t} w_{k}
$$

be the steady and transient parts of the solution.

Since the problem is positive, we have that $w_{1}, w_{2}>0$, and thus if $v_{0}>0$ we have $a_{1}, a_{2}>0$.

We also observe that

$$
\lim _{t \rightarrow \infty} \mathrm{e}^{\frac{\lambda_{3}}{2} t} v_{t}=0
$$

Hence, there exists a time $T$ such that $v(\cdot, t)>0$, for $t \geq T$. Let $t^{*} \geq 0$ be the minimal time such that $v(., t) \geq 0$, for $t \geq t^{*}$. Clearly $0 \leq t^{*}<T$.

Assume that $t^{*}>0$. Since $v$ is smooth, we have that

$$
\lim _{t \downarrow t^{*} \operatorname{int}(\mathrm{I})} v(\cdot, t)=0
$$

On the other hand, since the coefficients are bounded, we can assume without loss of generality that the strong maximum principle holds for $L$. Thus, the parabolic Harnack inequality—cf. [32,42]—holds for $t \geq t^{*}$, and together with Equation 11 yields that $v\left(\cdot, t^{*}\right)=0$.

Let $\phi_{1}$ be a positive conservation law, which exists since the problem is intrinsically positive. Then we have

$$
0=\left\langle v\left(\cdot, t^{*}\right), \phi_{1}\right\rangle=\left\langle v(\cdot, T), \phi_{1}\right\rangle>0,
$$

which is a contradiction. Therefore $t^{*}=0$, and the solution is positive. 
If $v_{0} \geq 0$, add a positive constant $K$ to $v_{0}$, and then by letting $K \rightarrow 0$ we obtain that $v(\cdot, t) \geq 0$ as claimed.

Finally, the result for $p, q \in L^{2}(I) \cap L^{\infty}(I)$ follows by a standard mollification argument.

For uniqueness, we first observe that, if $v_{0} \equiv 0$, then we must have $v(\cdot, t) \geq 0$, for all time. As before, let $\phi_{1}$ be a positive conservation law for (9). Then

$$
\left\langle v(\cdot, t), \phi_{1}\right\rangle=\left\langle v_{0}, \phi_{1}\right\rangle=0
$$

Therefore, we have $v(\cdot, t)=0$, and uniqueness follows.

We now want to briefly discuss the case when there is only one conservation law, namely:

Definition 3. A partially conservative problem is an initial value given by

$$
\left\{\begin{array}{lr}
\partial_{t} v=L v, & \text { in } I \text { and } t>0 \\
\frac{\mathrm{d}}{\mathrm{d} t}\left\langle v(\cdot, t), \phi_{1}\right\rangle=0, & t>0 \\
B\left(v(a, t), v(b, t), \partial_{x} v(a, t), \partial_{x} v(b, t)\right)=0, & \\
v(x, 0)=v_{0}(x) &
\end{array}\right.
$$

if $L \phi_{1}=0$, and the corresponding spectral problem is self-adjoint. We shall also say that (12) is a positive problem, if $\phi_{1}>0$.

Minor modifications of the previous arguments are necessary to prove the following:

Theorem 3. Consider the initial value problem (12) and assume that it is a positive partially conservative problem. If we have $v_{0} \in L^{2}(I)$, then, for any $T>0$, there exists a unique solution in the class $C\left([0, T) ; L^{2}(I)\right) \cap C^{\infty}\left((0, T) ; \mathcal{H}_{1}\right)$. If, in addition, $\partial_{t} v-L v$ satisfies the strong maximum principle, then for $v_{0} \geq 0$ we have that $v(\cdot, t) \geq 0$ for every $t$.

Remark 3. The heat equation with homogeneous Neumann conditions is a positive partially conservative problem. Indeed, one can take $\phi_{1}=1$, and as an additional boundary condition that the flux in one of the endpoints should vanish. In this case, while the existence and uniqueness are well-known, Theorem 3 
provides which seems to be a new alternative argument for positiveness that does not require knowledge of the boundary behaviour of the solution.

Remark 4. For the general problem

$$
\begin{cases}\partial_{t} u=M u, t>0, x \in(a, b) & \\ \frac{\mathrm{d}}{\mathrm{d} t}\left(u, \phi_{1}\right)=0, & t>0 \\ \frac{\mathrm{d}}{\mathrm{d} t}\left(u, \phi_{2}\right)=0, & t>0 \\ u(0, x)=u_{0}(x) . & \end{cases}
$$

with

$$
M u=a(x) \partial_{x}^{2} u+b(x) \partial_{x} u+c(x) u,
$$

and with the coefficient a bounded away from zero, we set

$$
\eta(x)=\exp \left(\int_{a}^{x} \frac{b(s)}{a(s)} \mathrm{d} s\right)
$$

Then, we can recast the problem as

$$
\begin{cases}\partial_{t} u=L u, t>0, x \in(a, b) & \\ \frac{\mathrm{d}}{\mathrm{d} t}\left(u, \psi_{1}\right)=0, & t>0 \\ \frac{\mathrm{d}}{\mathrm{d} t}\left(u, \psi_{2}\right)=0, & t>0 \\ u(0, x)=u_{0}(x) . & \end{cases}
$$

with

$$
L u=\frac{a}{\eta}\left[\partial_{x}\left(\eta \partial_{x} u\right)+\frac{c \eta}{a} u\right]
$$

and

$$
\psi_{i}=\frac{a}{\eta} \phi_{i}, \quad i=1,2 .
$$

Then all previous results apply, provided all the spaces are weighted with respect to the measure

$$
\mathrm{d} \mu=\frac{\eta}{a} \mathrm{~d} x
$$


Notice also that all inner products, inclusive the ones in the conservation laws, are now given with respect to the weighted measure $\mathrm{d} \mu$.

Remark 5. Conservative problems can be seen as a special case of the more general problem of prescribing the value of two functionals along the solution. Indeed, if one prescribes the linear moments to be constant, that are compatible with the initial conditions, one gets a conservative parabolic problem. More generally, by considering, without loss of generality, that $\phi_{1}$ and $\phi_{2}$ have unit norm and are orthogonal, we specify

$$
\left\langle v(\cdot, t), \phi_{i}\right\rangle=F_{i}(t), \quad i=1,2 .
$$

After writing

$$
w(x, t)=v(x, t)-F_{1}(t) \phi_{1}(x)-F_{2}(t) \phi_{2}(x),
$$

then $w$ satisfies a non-homogeneous conservative parabolic problem. Indeed, in this case we have

$$
\left\langle w(\cdot, t), \phi_{i}\right\rangle=0, \quad i=1,2 .
$$

and

$$
\partial_{t} w=L w+G(x, t), \quad G(x, t)=F_{1}^{\prime}(t) \phi_{1}(x)+F_{2}^{\prime}(t) \phi_{2}(x) .
$$

This last problem can then be solved using Duhamel principle.

\section{From degenerated to non-degenerated problems and back}

We are interested in dealing with Fokker-Planck equations of the following type

$$
\partial_{t} u=\partial_{x}^{2}(g u)-\partial_{x}(g \psi u)=0
$$

where we shall always assume that $g \in C^{\infty}([0,1])$, that $g(0)=0$ and that $u$ satisfies

$$
\frac{\mathrm{d}}{\mathrm{d} t} \int_{0}^{1} u(x, t) \mathrm{d} x=0
$$

We shall be interested in two different cases:

1. $g(1)=0$, and $u$ then further satisfies

$$
\frac{\mathrm{d}}{\mathrm{d} t} \int_{0}^{1} \varphi(x) u(x, t) \mathrm{d} x=0
$$


2. $g(1)>0$, and $u$ then further satisfies

$$
\partial_{x}(g u)-\left.g \psi u\right|_{x=1}=0
$$

\subsection{Elliptic self-adjoint perturbations}

Let $g_{\varepsilon}:[0,1] \rightarrow \mathbb{R}$ be a positive smooth function such that

$$
\lim _{\varepsilon \rightarrow 0} g_{\varepsilon}(x)=g(x), \quad \text { pointwise }
$$

and consider the $\varepsilon$-perturbed problem:

$$
\begin{aligned}
& u_{t}^{(\varepsilon)}=\left(g_{\varepsilon}(x) u^{(\varepsilon)}\right)_{x x}-\left(g_{\varepsilon}(x) \psi(x) u^{(\varepsilon)}\right)_{x}, \\
& \frac{\mathrm{d}}{\mathrm{d} t} \int u^{(\varepsilon)}(x, t) \mathrm{d} x=0, \\
& \frac{\mathrm{d}}{\mathrm{d} t} \int \varphi(x) u^{(\varepsilon)}(x, t) \mathrm{d} x=0, \\
& u^{(\varepsilon)}(x, 0)=u_{(\mathrm{I})}(x) .
\end{aligned}
$$

when $g(1)=0$. If $g(1)>0$ we then replace (19) by

$$
\partial_{x}\left(g_{\varepsilon}(x) u^{(\varepsilon)}(1, t)\right)-\left.g_{\varepsilon}(x) \psi(x) u^{(\varepsilon)}(x, t)\right|_{x=1}=0 .
$$

This problem is now amenable to be treated using the ideas developed in Section 2. In order to write the problem in self-adjoint form and to obtain boundary conditions that are independent of $\varepsilon$ we introduce the following change of variables:

$$
u^{(\varepsilon)}(x, t)=\frac{v^{(\varepsilon)}(x, t)}{g_{\varepsilon}(x)} p(x), \quad p(x)=\exp \left(\int_{0}^{x} \psi(y) \mathrm{d} y .\right) .
$$

In this new variable, we can apply Theorem 1 and then the corresponding formulation given in (10) for equations (17), (18), (19), and (20) becomes, respectively,

$$
\begin{aligned}
v_{t}^{(\varepsilon)} & =\frac{g_{\varepsilon}}{p}\left(p v_{x}^{(\varepsilon)}\right)_{x} \\
v_{x}^{(\varepsilon)}(1, t) p(1) & =v_{x}^{(\varepsilon)}(0, t) \\
v_{x}^{(\varepsilon)}(1, t) p(1)-\varphi^{\prime}(1) v^{(\varepsilon)}(1, t) p(1) & =-\varphi^{\prime}(0) v(0, t) \\
v_{(\mathrm{I})}^{(\varepsilon)} & :=v^{(\varepsilon)}(x, 0)=u_{(\mathrm{I})} \frac{g_{\varepsilon}}{p} .
\end{aligned}
$$

provided $g(1)=0$. If $g(1)>0$, we replaced (24) by

$$
\partial_{x} v^{(\varepsilon)}(1, t)=0 .
$$

Theorem 2 applied to (22), (23),(24) and (25) or applying Theorem 3 with (24) replaced by (26) yields the following result: 
Proposition 1. Let $u_{(\mathrm{I})} \in \mathcal{B M}^{+}([0,1])$ and assume that $\psi \in L^{1}((0,1), \mathrm{d} \mu)$. Then we have that (22), (23) and (24) or (26)) has a unique solution in the class $C\left([0, \infty) ; \mathcal{B M}^{+}([0,1])\right) \cap C^{1}\left([0, \infty) ; H^{1}((0,1))\right)$. Moreover, this solution can be written as

$$
v^{(\varepsilon)}=\sum_{k=0}^{\infty} a_{k} \mathrm{e}^{-\lambda_{k} t} w_{k}, \quad a_{k}=\left(v_{(\mathrm{I})}^{(\varepsilon)}, w_{k}\right)
$$

In addition, if $v_{(\mathrm{I})}^{(\varepsilon)}>0$, then $v^{(\varepsilon)}(\cdot, t)>0$.

Remark 6. If $\psi$ is continuous then the eigenfunctions $w_{k}$ are $C^{2}$, and hence we have that $v^{(\varepsilon)}$ is a $C^{2}$ classical solution for $t>0$.

\subsection{The vanishing perturbation limit}

Since $v^{(\varepsilon)}$ are positive we have also that $u^{(\varepsilon)}$ are positive, and because of conservation law (14) they can be seen as Radon measures with a fixed given mass. In this case, Prohorov's theorem — cf. [2] — implies that we have a (subsequencewise) limit as $\varepsilon$ goes to zero. Namely,

Proposition 2. Fix $T>0$ and let $\mathcal{C}(T)=[0,1] \times[0, T]$. Then, by passing a subsequence if necessary, we can assume that $u^{(\varepsilon)} \rightarrow u^{(0)}$ such that $u^{(0)} \in \mathcal{B M}^{+}(\mathcal{C}(T))$.

Remark 7. First, it is important to observe that we cannot interchange the limits $T \rightarrow \infty$ and $\varepsilon \rightarrow 0$. Indeed, from the solution in the proof of Proposition 1 we have that $\lim _{t \rightarrow \infty} u^{(\varepsilon)}(\cdot, t)$ is nonzero, regular and independent of $\varepsilon$, since 1 and $\varphi$ are independent of $\varepsilon$. On the other hand, we shall see in Section 4 that the large time limit of $u^{(0)}$ is a linear combination of atomic measures supported at the boundaries.

We now obtain a weak equation in weak form for the limiting measure. Let

$$
\Gamma:=C_{c}^{1}([0, \infty) ; \mathcal{D}),
$$

where

$$
\mathcal{D}=\left\{\eta \in C^{2}((0,1)) \cap C^{1}([0,1]) \text { such that }(23) \text { and }(24) \text { are satisfied }\right\} .
$$

The corresponding weakest formulation of (22) is given by

$-\int_{0}^{\infty} \int_{0}^{1} v^{(\varepsilon)}(x, t) \frac{p(x)}{g_{\varepsilon}(x)} \partial_{t} \alpha(x, t) \mathrm{d} x \mathrm{~d} t-\int_{0}^{1} v^{(\varepsilon)}(x, 0) \frac{p(x)}{g_{\varepsilon}(x)} \alpha(x, 0) \mathrm{d} x=\int_{0}^{\infty} \int_{0}^{1} v^{(\varepsilon)}(x) \partial_{x}\left(p(x) \partial_{x} \alpha(x, t)\right) \mathrm{d} x \mathrm{~d} t$,

with $\alpha \in \Gamma$. 
Using the relationship between $u^{(\varepsilon)}$ and $v^{(\varepsilon)}$, the definition of $\mathrm{d} \mu$ and that $p^{\prime}=\psi p$ we obtain $-\int_{0}^{\infty} u^{(\varepsilon)}(x, t) \partial_{t} \alpha(x, t) \mathrm{d} x \mathrm{~d} t-\int_{0}^{1} u^{(\varepsilon)}(x, 0) \alpha(x, 0) \mathrm{d} x=\int_{0}^{\infty} \int_{0}^{1} u^{(\varepsilon)}(x) g_{\varepsilon}(x)\left(\partial_{x}^{2} \alpha(x, t)+\psi(x) \partial_{x} \alpha(x, t)\right) \mathrm{d} x \mathrm{~d} t$.

Now, we consider the limit $\varepsilon \rightarrow 0$ :

Proposition 3. The limiting measure $u^{(0)}$ is in the class $L^{\infty}\left([0, \infty) ; \mathcal{B M}^{+}([0,1])\right)$, and it satisfies

$$
\begin{aligned}
-\int_{0}^{\infty} & \int_{0}^{1} u^{(0)}(x, t) \partial_{t} \alpha(x, t) \mathrm{d} x \mathrm{~d} t-\int_{0}^{1} u^{(0)}(x, 0) \alpha(x, 0) \mathrm{d} x \\
= & \int_{0}^{\infty} \int_{0}^{1} u^{(0)}(x, t) g(x)\left(\partial_{x}^{2} \alpha(x, t)+\psi(x) \partial_{x} \alpha(x, t)\right) \mathrm{d} x \mathrm{~d} t,
\end{aligned}
$$

with test functions $\alpha \in \Gamma$. In addition, it satisfies the conservation laws (14) and (15), when $g(1)=0$ and the conservation law (15) and the boundary condition (16), when $g(1)>0$.

Proof. Convergence follows from standard arguments; [2]. When $g(1)=0$, it remains only to show the conservation laws. This can be done either by appealing to standard convergence theorems and taking the limits in (18) and (19) or, if $\psi$ is at least continuous, as in [13], by considering test functions of the form

$$
\alpha(x, t)=\beta(t) \gamma(x), \quad \beta(t) \in C_{c}^{1}((0, \infty)), \quad \gamma \in \operatorname{span}\{1, \varphi\} .
$$

When $g(1)>0$, the conservation law (14) is verified analogously. The boundary condition (16) can be verified by first observing that the solution has to be smooth near $x=1$. This can be seen by considering test functions with compact support in $(1 / 2,1] \times(0, T]$, and then applying local parabolic regularity. Integration by parts then yields the result.

Remark 8. Notice that regardless of the regularity of $\psi$, we always have that 1 is in $\mathcal{D}$. On the other hand, we have $\varphi \in \mathcal{D}$ if, and only if, $\psi$ is continuous.

\section{Further properties of the weak solution}

We now proceed to understand what properties the solution to (27) possesses. In particular, when $g(1)=0$, we show uniqueness and that the solution found here is the same as the one found in [14] where the test space is taken to be $C_{c}^{1}\left([0, \infty) ; C^{2}([0,1])\right)$. 
Before we can state our results, we need a decomposition result for compact distributions in [14], which we recall for the convenience of the reader.

Lemma 2 (Decomposition). Denote by $\mathcal{E}^{\prime}$ the space of compactly supported distributions in $\mathbb{R}$. Let $\nu \in \mathcal{E}^{\prime}$ with $\operatorname{sing} \operatorname{supp}(\nu) \subset[0,1]$. Then the setwise decomposition

$$
[0,1]=\{0\} \cup(0,1) \cup\{1\}
$$

yields a decomposition in $\nu$, namely

$$
\nu=\nu_{0}+\mu+\nu_{1}
$$

where $\nu_{i}$ is a compact distribution supported at $x=\{i\}$, and we also have that $\operatorname{sing} \operatorname{supp}(\mu) \subset(0,1)$. Moreover, if $\nu$ is a Radon measure, then $\mu \in \mathcal{B M}((0,1))$ and $\nu_{i}=c_{i} \delta_{i}$, where $\delta_{i}$ are normalised atomic measures with support in $x=\{i\}$.

We are now ready to discuss the two classes of examples that we are considering here.

\subsection{The generalised Kimura equation}

The main result is then as follows

Theorem 4. Let $\mathfrak{D}$ be a domain such that $C_{c}^{2}((0,1)) \oplus \operatorname{span}(\{1\}) \subset \mathfrak{D}$, and consider $(27)$, with test functions in $\mathfrak{D}$ and initial condition $u_{(\mathrm{I})} \in \mathcal{B M}^{+}([0,1])$. Applying the decomposition in Lemma 2, we can write

$$
u_{(\mathrm{I})}=a_{0} \delta_{0}+r_{0}+b_{0} \delta_{1}, \quad a_{0}, b_{0}, r_{0} \geq 0
$$

Then any solution to (27) can be represented as

$$
u^{(0)}(\cdot, t)=a(t) \delta_{0}+r+b(t) \delta_{1}
$$

where $r$ is the unique strong solution to (1) without any boundary condition and initial condition $r_{0}$, and $a, b:[0, \infty) \rightarrow[0, \infty)$ are smooth. Furthermore, we have that

$$
\begin{aligned}
& a(t)=a_{0}+\int_{0}^{1} r_{0}(x)(1-\varphi(x)) \mathrm{d} x-\int_{0}^{1} r(x, t)(1-\varphi(x)) \mathrm{d} x \\
& b(t)=b_{0}+\int_{0}^{1} u_{(\mathrm{I})}(x) \varphi(x) \mathrm{d} x-\int_{0}^{1} r(x, t) \varphi(x) \mathrm{d} x .
\end{aligned}
$$


In particular, we have

$$
u^{(0)} \in C^{\infty}\left((0, \infty) ; H^{1}\right) \cap C^{0}\left([0, \infty) ; \mathcal{B} \mathcal{M}^{+}\right),
$$

and that $u^{(0)}$ is unique in this class.

Proof. Notice that $C_{c}^{2}((0,1)) \subset \mathfrak{D}$. Hence, by restricting to that domain we obtain the weak formulation to (1) without any boundary condition, and initial condition given by $r_{0}$. This equation is known to be well posed and to have a strong solution in $C^{1}\left((0, \infty) ; H^{1}(0,1)\right) \cap C^{0}\left([0, \infty) ; \mathcal{B M}^{+}\right)$.

By applying the decomposition in $u^{(0)}$ together with (27) and still restricting to test functions in $C_{c}^{2}((0,1))$, we conclude that the non-atomic part of $u^{(0)}$ must be $r$.

Since $u^{(0)}$ is a Radon measure, Lemma 2 yields

$$
u^{(0)}(\cdot, t)=a(t) \delta_{0}+r(\cdot, t)+b(t) \delta_{1} .
$$

The formulas for $a$ and $b$ follow from direct substitution of (29) in the integrated forms of (3) and (4).

Remark 9. In particular, we have that the solution is unique, and that it does not depend on the particular domain $\mathfrak{D}$-provided it satisfies the required condition. Notice that both $\mathcal{D}$ and $C_{0}^{1}\left([0, \infty) ; C^{2}([0,1])\right.$ both satisfy this requirement, and hence the solution obtained from the elliptical perturbations is the same as the one obtained in [14]. In particular, the asymptotic limit in time is given by

$$
u_{\infty}^{(0)}(x)=a_{\infty} \delta_{0}+b_{\infty} \delta_{1}, \quad\left(a_{\infty}, b_{\infty}\right):=\lim _{t \rightarrow \infty}(a(t), b(t)) .
$$

Further regularity in $\psi$ allows for a more detailed description of $a$ and $b$ :

Theorem 5. In the same framework of Theorem 4 assume, in addition, that $\psi$ is continuous and that $\mathfrak{D} \supset C_{c}^{2}((0,1)) \oplus \operatorname{span}(\{1, \varphi\})$. Then we have that

$$
u^{(0)} \in C^{\infty}\left((0, \infty) ; C^{2}([0,1])\right) \cap C^{0}\left([0, \infty) ; \mathcal{B M}^{+}([0,1])\right) .
$$

Moreover, we have that

$$
a(t)=a_{0}+\int_{0}^{t} r(0, s) \mathrm{d} s \quad \text { and } \quad b(t)=b_{0}+\int_{0}^{t} r(1, s) \mathrm{d} s .
$$


Proof. The extra regularity follows from the improved regularity of the eigenfunctions - cf. Remark 6 . Then direct substitution of (29) in (27), and by taking advantage of the improved regularity of $r$ to integrate by parts yields

$$
\int_{0}^{\infty} a(t) \partial_{t} \alpha(0, t) \mathrm{d} t+a_{0} \alpha(0,0)+b_{0} \alpha(1,0)+\int_{0}^{\infty} r(0, t) \alpha(0, t) \mathrm{d} t+\int_{0}^{\infty} r(1, t) \alpha(1, t) \mathrm{d} t=0 .
$$

Choosing test functions that vanish at either endpoint then finishes the proof.

\subsection{The SIS-PDE model}

Now, we return to the SIS-PDE model (6)-(8). Let

$$
F(x)=R_{0}(1-x)+1, \quad \text { and } \quad H(x)=x+\frac{2}{R_{0}} \log \left(\frac{F(x)}{F(0)}\right) .
$$

Also let

$$
\omega(x)=\frac{P(x)}{x F(x)}, \quad P(x)=\exp (2 H(x))
$$

We shall also write

$$
\omega^{\epsilon}=\frac{P(x)}{(x+\epsilon) F(x)} \quad \text { and } \quad v^{(\varepsilon)}(x, t)=\frac{p(x, t)}{\omega^{\epsilon}(x)}
$$

Then, replacing the conservation law by the corresponding non-local boundary condition, we obtain

$$
\begin{aligned}
& \partial_{t} v^{(\varepsilon)}=\frac{1}{2 \omega^{\epsilon}(x)} \partial_{x}\left(P(x) \partial_{x} v^{(\varepsilon)}\right) \\
& P(1) \partial_{x} v^{(\varepsilon)}(1, t)-P(0) \partial_{x} v^{(\varepsilon)}(0, t)=0 \\
& \partial_{x} v^{(\varepsilon)}(1, t)=0 \\
& v^{(\varepsilon)}(x, 0)=\frac{1}{\omega^{\epsilon}(x)} p_{(\mathrm{I})}(x)
\end{aligned}
$$

Theorem 3 then immediately translates into the following result:

Theorem 6. For each $\epsilon>0$, Equation (30) has a unique solution, that is positive (non-negative) if $p_{(\mathrm{I})}$ is positive (non-negative). We also have that $v^{(\varepsilon)} \rightarrow v^{(0)}$ weakly in $[0,1]$, as $\epsilon \rightarrow 0$. Let $p^{(0)}$ be the limiting 
solution in the original variables. Then it satisfies:

$$
\begin{aligned}
& \int_{0}^{\infty} \int_{0}^{1} p^{(0)}(x, t) \partial_{t} \phi(x, t) \mathrm{d} x \mathrm{~d} t \\
& \quad+\frac{1}{2} \int_{0}^{\infty} \int_{0}^{1} p^{(0)}(x, t) x\left(R_{0}(1-x)+1\right) \partial_{x}^{2} \phi(x, t) \mathrm{d} x \mathrm{~d} t \\
& \quad+\int_{0}^{\infty} \int_{0}^{1} p^{(0)}(x, t) x\left(R_{0}(1-x)-1\right) \partial_{x} \phi(x, t) \mathrm{d} x \mathrm{~d} t \\
& \quad+\int_{0}^{1} p^{(0)}(x, 0) \phi(x, 0) \mathrm{d} x=0 .
\end{aligned}
$$

In particular it satisfies the conservation law (8), and it can be written as

$$
p^{(0)}(t, x)=a(t) \delta_{0}+r(x, t), \quad a(t)=\frac{R_{0}+1}{2} \int_{0}^{t} r(0, s) \mathrm{d} s+a_{0},
$$

and where $r$ satisfies equation (6) with the boundary condition

$$
\frac{1}{2}\left(\left(1-R_{0}\right) r(1, t)+\partial_{x} r(1, t)\right)+r(1, t)=0 .
$$

\section{Discussion}

This work is a first systematic step into the theory of conservative and degenerated parabolic problems. These problems appear in a number of modelling situations, as discussed in Section 1. We restrict ourselves to $1+1$ problems, and begin by presenting the theory for the case of uniformly parabolic infinitesimal generators in Section 2, which provides a comprehensive formulation for such problems from the point of view of SturmLiouville theory. As a by-product of the analysis, we also present a proof of persistence of positiveness that seems to be new even for the traditional Neumann problem for the heat equation, since it does not require the study of the solution at the boundaries. The results described in this section can be seen as an alternative approach to [44] that naturally extends to equations with non-constant coefficients - albeit not easily for higher dimensions. They also show how some non-local problems can be brought into a framework that is similar to the treatment of more classical boundary value problems.

If the operator is degenerated at at least one of the endpoints then two situations can arise: if the operator is self-adjoint, the analysis goes through unchanged. Otherwise, although one can still bring the problem into self-adjoint form, it is possible that the solution in the original variables will not be integrable. An example of this situation is the generalised Kimura equation (1), and this is studied in sections 3.1 and 4.1. Indeed, when working in self-adjoint variables for this problem, one needs to enforce homogeneous Dirichlet conditions which, in turn, yield a negative-definite problem - hence no conservation seems possible. However, by a perturbation argument we can apply the results of Section 2, and by considering positive solutions and the weakest formulation for the problem, we can take the limit of vanishing perturbation in measure space, and obtain a measure solution for the original equation. This was discussed in section 3.2.

In section 4.2, we presented an example of a recently derived Fokker-Plank equation (6) that arises from an epidemiological problem, and that is half-degenerated, supplemented by one conservation law. The 
technique used to obtain measure solutions of this problem is similar to the one described previously in this section.

The degenerated examples presented already suggest that a very natural extension of this work is to study in more detail the endpoint degenerated cases. The aim would be to provide a complete classification of these problems, when the infinitesimal generator is not self-adjoint.

Another natural extension is to study the case in more than one spatial variable. The analysis in Section 2 made extensive use of 1-d Sturm-Liouville theory, the generalisation to more dimensions is far from straightforward. One example of interest is the generalised Kimura equation obtained in [16] (more restricted examples appear in $[29,33])$. A second possible example of interest, that generalizes the SISPDE studied in this work, is the SIR-PDE (where a third class of individuals, the Removed individuals is considered). Although the derivation is not complete, some preliminary results were presented in [15].

\section{Acknowledgements}

OD: Supported by the Erasmus Mundus Action 2 Programme of the European Union, and Universidade Nova de Lisboa.

MOS: Partially supported by CNPq under grant \# 308113/2012-8 and acknowledge the hospitality of $\mathrm{CMA} / \mathrm{FCT} / \mathrm{UNL}$.

FACCC: Partially supported by FCT/Portugal Strategic Project UID/MAT/00297/2013 (Centro de Matemática e Aplicações, Universidade Nova de Lisboa) and by a "Investigador FCT" grant.

\section{References}

[1] P. Bailey, W. Everitt, and A. Zettl. Regular and singular Sturm-Liouville problems with coupled boundary conditions. P. Roy. Soc. Edinb. A, 126(03):505-514, 1996. DOI: 10.1017/S0308210500022885

[2] P. Billingsley. Convergence of Probability Measures. John Wiley \& Sons, New York, 1999.

[3] A. Bobrowski and D. Mugnolo. On moments-preserving cosine families and semigroups in $C[0,1]$. $J$. Evol. Equ., 13(4):715-735, 2013. DOI: 10.1007/s00028-013-0199-x

[4] A. Bouziani. Mixed problem with boundary integral conditions for a certain parabolic equation. Int. J. Stoch. Anal., 9(3):323-330, 1996. DOI: 10.1155/S1048953396000305

[5] A. Bouziani. Strong solution for a mixed problem with nonlocal condition for certain pluriparabolic equations. Hiroshima Math. J., 27(3):373-390, 1997.

[6] A. Bouziani. On the solvability of parabolic and hyperbolic problems with a boundary integral condition. Int. J. Math. Math. Sci., 31(4):201-213, 2002. DOI: 10.1155/S0161171202005860

[7] A. Bouziani and N. Benouar. Probleme mixte avec conditions integrales pour une classe d'equations hyperboliques. B. Belg. Math. Soc.-Sim., 3(2):137-145, 1996.

[8] A. Bouziani, N. Merazga, and S. Benamira. Galerkin method applied to a parabolic evolution problem with nonlocal boundary conditions. Nonlinear Anal.-Theor., 69(5-6):1515 - 1524, 2008. DOI: 10.1016/j.na.2007.07.008 
[9] J. Cannon. The solution of the heat equation subject to the specification of energy. Quart. Appl. Math, 21(2):155-160, 1963.

[10] J. Cannon and J. van der Hoek. The classical solution of the one-dimensional two-phase Stefan problem with energy specification. Ann. Mat. Pur. Appl., 130(1):385-398, 1982.

[11] J. R. Cannon and J. van der Hoek. Diffusion subject to the specification of mass. J. Math. Anal. Appl., 115(2):517 - 529, 1986. DOI: 10.1016/0022-247X(86)90012-0

[12] Fabio A. C. C. Chalub and Max O. Souza. Discrete versus continuous models in evolutionary dynamics: from simple to simpler-and even simpler-models. Math. Comput. Modelling 47(7-8):743-754, 2008. DOI: $10.1016 /$ j.mcm.2007.06.009

[13] F. A. C. C. Chalub and M. O. Souza. From discrete to continuous evolution models: A unifying approach to drift-diffusion and replicator dynamics. Theor. Pop. Biol., 76(4):268-277, 2009. DOI: 10.1016/j.tpb.2009.08.006

[14] F. A. C. C. Chalub and M. O. Souza. A non-standard evolution problem arising in population genetics. Commun. Math. Sci., 7(2):489-502, 2009.

[15] F. A. C. C. Chalub and M. O. Souza. The SIR epidemic model from a PDE point of view. Math. Comput. Model., 53(7-8):1568-1574, 2011. DOI: 10.1016/j.mcm.2010.05.036

[16] F. A. C. C. Chalub and M. Souza. The frequency-dependent Wright-Fisher model: diffusive and nondiffusive approximations. J. Math. Biol., 68(5):1089-1133, 2014. DOI: 10.1007/s00285-013-0657-7

[17] F. A. C. C. Chalub and M. O. Souza. Discrete and continuous SIS epidemic models: A unifying approach. Ecol. Complex., 18:83 - 95, 2014. DOI: 10.1016/j.ecocom.2014.01.006

[18] N. Champagnat, R. Ferrière and S. Méléard, Unifying evolutionary dynamics: from individual stochastic processes to macroscopic models. Theor. Popul. Biol. 69(3):297-321, 2006. DOI: 10.1016/j.tpb.2005.10.004

[19] E. A. Coddington and N. Levinson. Theory of Ordinary Differential Equations. McGraw Hill, 1955.

[20] C. M. Dafermos. Hyperbolic conservation laws in continuum physics, volume 325 of Grundlehren der Mathematischen Wissenschaften. Springer-Verlag, Berlin,, 2005.

[21] D.-Q. Dai and Y. Huang. Remarks on a semilinear heat equation with integral boundary conditions. Nonlinear Anal.-Theor., 67(2):468 - 475, 2007. DOI: 10.1016/j.na.2006.06.012

[22] K. Deckert and C. Maple. Solutions for diffusion equations with integral type boundary condition. In Proc. Iowa Acad. Sc, volume 70, pages 354-361, 1963.

[23] M. Dehghan. Numerical solution of a parabolic equation with non-local boundary specifications. Appl. Math. Comput., 145(1):185 - 194, 2003. DOI: 10.1016/S0096-3003(02)00479-4

[24] M. Dehghan. Numerical solution of a parabolic equation subject to specification of energy. Appl. Math. Comp. 149: 31-45. 2004. DOI: 10.1016/S0096-3003(02)00954-2

[25] M. Dehghan. Application of the Adomian decomposition method for two-dimensional parabolic equation subject to nonstandard boundary specifications. Appl. Math. Comp 157:549-560, 2004. DOI: 10.1016/j.amc.2003.08.098 
[26] M. Dehghan. The one-dimensional heat equation subject to a boundary integral specification. Chaos Soliton. Fract. 32: 661-675, 2007. DOI: 10.1016/j.chaos.2005.11.010

[27] E. DiBenedetto. Degenerate Parabolic Equations. Springer-Verlag, 1993.

[28] P. G. Drazin, R. S. Johnson. Solitons: An Introduction. Cambridge University Press, 1989.

[29] C. E. Epstein and R. Mazzeo. Degenerate diffusion operators arising in population biology. Princeton, NJ: Princeton University Press, 2013.

[30] S. N. Ethier. A class of degenerate diffusion processes occurring in population genetics. Comm. Pure Appl. Math., 29(5):483-493, 1976. DOI: 10.1002/cpa.3160290503

[31] S. N. Ethier and T. G. Kurtz. Markov processes: Characterization and convergence. Wiley Series in Probability and Mathematical Statistics: Probability and Mathematical Statistics, John Wiley \& Sons Inc., New York, 1986, .

[32] L. C. Evans. Partial differential equations, volume 19 of Graduate Studies in Mathematics. American Mathematical Society, Providence, RI, 1998.

[33] W. J. Ewens. Mathematical Population Genetics. I, volume 27 of Interdisciplinary Applied Mathematics. Springer-Verlag, New York, Second edition, 2004. Theoretical introduction.

[34] W. Feller. Diffusion processes in genetics. In Proceedings of the Second Berkeley Symposium on Mathematical Statistics and Probability, 1950, pages 227-246, Berkeley and Los Angeles, 1951. University of California Press.

[35] A. Friedman. Partial differential equations of parabolic type. Courier Dover Publications, 2013.

[36] N. I. Ionkin. Solution of a boundary-value problem in heat conduction with a nonclassical boundary condition. Diff. Equat., 13, 204-211 (1977). Translated from Differ. Uravn 13:294-304, 1977. DOI: 10.1016/0041-5553(64)90080-1

[37] N. I. Ionkin and E.I. Moiseev. A problem for the heat conduction equation with two-point boundary condition. Differ. Equations 15, 915-923, 1980. Translated from Differ. Uravn, 15:1284-1295, 1979.

[38] F. John. Partial differential equations, (4th ed.). New York: Springer-Verlag, 1982.

[39] L. Kamynin. A boundary value problem in the theory of heat conduction with a nonclassical boundary condition. USSR Comp. Math. Math., 4(6):33 - 59, 1964. DOI: 10.1016/0041-5553(64)90080-1

[40] M. Kimura. On the probability of fixation of mutant genes in a population. Genetics, 47:713-719, 1962.

[41] Q. Kong and A. Zettl. Dependence of eigenvalues of Sturm-Liouville problems on the boundary. J. Differ. Equations, 126(2):389-407, 1996. DOI: 10.1006/jdeq.1996.0056

[42] G. M. Lieberman. Second order parabolic differential equations. World Scientific Publishing Co. Inc., River Edge, NJ, 1996.

[43] D. Mugnolo and S. Nicaise. Diffusion processes on an interval under linear moment conditions. Semigroup Forum, 88(2):479-511, 2014. DOI: 10.1007/s00233-013-9552-1

[44] D. Mugnolo and S. Nicaise. Well-posedness and spectral properties of heat and wave equations with non-local conditions. J. Differ. Equations, 256(7):2115-2151, 2014. DOI: 10.1016/j.jde.2013.12.016 
[45] R. O. Popovych, M. Kunzinger and N. M. Ivanova. Conservation Laws and Potential Symmetries of Linear Parabolic Equations. Acta Appl Math, 100:113-185, 2008. DOI: 10.1007/s10440-007-9178-y

[46] L. Pul'kina. On a certain nonlocal problem for a hyperbolic equation. J. Math. Sci., 144(1):3832-3840, 2007. DOI: $10.1007 / \mathrm{s} 10958-007-0236-9$

[47] L. S. Pul'kina. Certain nonlocal problem for a degenerate hyperbolic equation. Math. Notes, 51(3):286290, 1992. DOI: 10.1007/BF01206393

[48] P. Shi. Weak solution to an evolution problem with a nonlocal constraint. SIAM J. Math. Anal., 24(1):46-58, 1993. DOI: $10.1137 / 0524004$

[49] M. E. Taylor. Partial differential equations. I, volume 115 of Applied Mathematical Sciences. Basic theory. Springer-Verlag, New York, 1996.

[50] J. L. Vázquez. The Porous Medium Equation: Mathematical Theory. Oxford Math. Monogr. Oxford Univ. Press, Oxford, 2007

[51] J. Weidmann. Spectral theory of ordinary differential operators. Springer-Verlag New York, Inc., 1987.

[52] G. Yang. Global well-posedness for the diffusion equation of population genetics. J. Math. Anal. Appl., 415(2):760 - 778, 2014. DOI: 10.1016/j.jmaa.2014.01.045

[53] N. I. Yurchuk. Mixed problem with an integral condition for certain parabolic equations. Diff. Equat., 22:1457-1463, 1986. Translated from Differ. Uravn. 22, No.12, 2117-2126 (1986).

[54] A. Zettl. Sturm-Liouville theory, volume 121 of Mathematical Surveys and Monographs. American Mathematical Society, Providence, RI, 2005. 